\title{
Multirate Hybrid CT/DT Cascade $\Sigma \Delta$ Modulators with Decreasing OSR of Back-end DT stages
}

\author{
J. Gerardo García-Sánchez and José M. de la Rosa \\ Instituto de Microelectrónica de Sevilla, IMSE-CNM (CSIC/University of Sevilla) \\ C/ Américo Vespucio s/n, 41092, Sevilla, SPAIN \\ Phone: + 34954466666, E-mail: jrosa@imse.cnm.es
}

\begin{abstract}
This paper presents novel architectures of multirate hybrid cascade continuous-time/discrete-time $\Sigma \Delta$ modulators that take advantage of the potentially faster operation of the continuoustime part of the circuit, while keep a reduced sampling operation of the back-end discrete-time stages. Compared to conventional multirate $\Sigma \Delta$ modulators, the proposed architectures use a higher sampling rate in the front-end (continuous-time) stage of the modulator, whereas the back-end (discrete-time) stages operate at a lower rate. It is demonstrated that the intrinsic aliasing signal can be cancelled in the digital domain, with no additional analog hardware required. The resulting $\Sigma \Delta$ topologies are potentially faster than conventional multirate $\Sigma \Delta$ modulators, more power efficient than hybrid monorate architectures and more robust than cascade continuous-time implementations. The combination of these features results in a new class of $\Sigma \Delta$ modulators, very suited for the implementation of analog-to-digital converters in the next generation of broadband wireless telecom systems.
\end{abstract}

\section{INTRODUCTION}

$\Sigma \Delta$ Modulators $(\Sigma \Delta \mathrm{Ms})$ have been commonly used in the last years for the implementation of Analog-to-Digital Converters (ADCs) in a number of telecom systems implemented in mainstream nanometer CMOS technologies. Although Discrete-Time (DT) implementations - normally using Switched-Capacitor (SC) circuits have been used by most reported state-of-the-art Integrated Circuits (ICs), the need of higher and higher data rates demands for unaffordable dynamic requirements of the operational amplifiers, what forces using low values of the OverSampling Ratio (OSR) - one of the main ingredients contributing to the performance of $\Sigma \Delta \mathrm{Ms}$ [1]. Alternatively, Continuous-Time (CT) $\Sigma \Delta$ Ms can provide faster rates than their DT counterparts with relatively lower power consumption. However, CT- $\Sigma \Delta \mathrm{Ms}$ are more sensitive than DT- $\Sigma \Delta \mathrm{Ms}$ against some circuit non-idealities, such as element tolerances and clock jitter [2].

The mentioned limitations can be partially solved by using the so-called Hybrid CT/DT $\Sigma \Delta \mathrm{Ms}(\mathrm{H}-\Sigma \Delta \mathrm{Ms})$, in which some DT parts of the $\Sigma \Delta \mathrm{M}$ - usually the front-end blocks - are replaced with CT circuits, thus providing faster operation and embedded anti-aliasing filtering, with reduced power consumption [3]-[10]. However, in spite of the mentioned advantages, the majority of reported $\mathrm{H}-\Sigma \Delta \mathrm{M}$ ICs [5]-[10] do not really exploit the capability of CMOS CT circuits to operate up to the $\mathrm{GHz}$ range with reasonable linearity [11], and hence to significantly increase the digitized signal bandwidth with medium-high values of OSR. This is due to the fact that the

This work has been supported by the Spanish Ministry of Science and Innovation (with support from the European Regional Development Fund) under contract TEC2007-67247-C02-01/MIC, and the Regional Ministry of Innovation, Science and Enterprise under contract TIC-2532. maximum sampling rate of $\mathrm{H}-\Sigma \Delta \mathrm{Ms}$ is indeed limited by the DT part of the system. A possible solution to alleviate this restriction could be using a different sampling frequency for each part (either CT or DT) of the $\mathrm{H}-\Sigma \Delta \mathrm{M}$, i.e applying multirating [12].

The idea of using MultiRate $\Sigma \Delta \mathrm{Ms}$ (MR- $\Sigma \Delta \mathrm{Ms}$ ) is not new and has been applied to both DT- and CT- $\Sigma \Delta M s$ [12][13]. However, in both cases, the strategy consists of using a lower OSR in the frontend blocks - where most of the power is consumed - and a higher OSR in the subsequent stages or blocks of the modulator - where the dynamic requirements can be relaxed. This approach has been recently applied to cascade $\mathrm{H}-\Sigma \Delta \mathrm{Ms}$, by using double-sampling technique to implement the DT back-end stage more efficiently [14].

This paper presents a new concept of multirate cascade $\Sigma \Delta \mathrm{Ms}$ that benefits of using a hybrid CT/DT implementation. The proposed cascade $\mathrm{H}-\Sigma \Delta \mathrm{Ms}$ use a higher OSR in the $\mathrm{CT}$ front-end stage whereas the remaining parts of the modulator - implemented using DT circuits - operate at lower values of OSR, thus relaxing the speed requirements of the corresponding $\mathrm{SC}$ integrators. The main drawback of this approach is that the downsampling operation of the back-end stages causes aliasing, what forces using an inter-stage Anti-Aliasing Filter (AAF). However, as demonstrated in this paper, the operation of the AAF can be translated into the digital domain, thus simplifying the analog part of the circuit. As a result, innovative multirate cascade $\mathrm{H}-\Sigma \Delta \mathrm{Ms}$ are synthesized, that can potentially achieve higher sampling rates than previously reported ones with no additional analog hardware required.

\section{Conventional MultiRate Cascade $\mathrm{H}-\Sigma \Delta \mathrm{MS}$}

Fig. 1 shows the conceptual implementation of a cascade 2-stage ${ }^{1}$ MultiRate Hybrid $\Sigma \Delta \mathrm{M}$ (MR $\mathrm{H}-\Sigma \Delta \mathrm{M})$. The circuit nature of the different modulator blocks, either CT or DT, as well as their corresponding sampling frequencies, $f_{\mathrm{si}}$, are highlighted in the figure. The most common situation is that the front-end stage - operating at $f_{\mathrm{s} 1}-$ is implemented using CT circuits, whereas the remaining $i$-th stages - sampled at $f_{\mathrm{si}}>f_{\mathrm{s} 1}-$ are based on DT circuits. The use of a complete CT stage (instead of just the front-end integrator) maximizes the embedded AAF [9], whereas takes advantage of both the relaxed dynamic requirements of the front-end CT circuitry as compared to their DT counterparts, and benefits from increasing

${ }^{1}$ For the sake of simplicity, the architectures under study in this paper focus on 2-stage cascade topologies. However, as will be shown in Section III, this analysis can be extended to more general cascade $N$-stage $\Sigma \Delta \mathrm{Ms}$. 


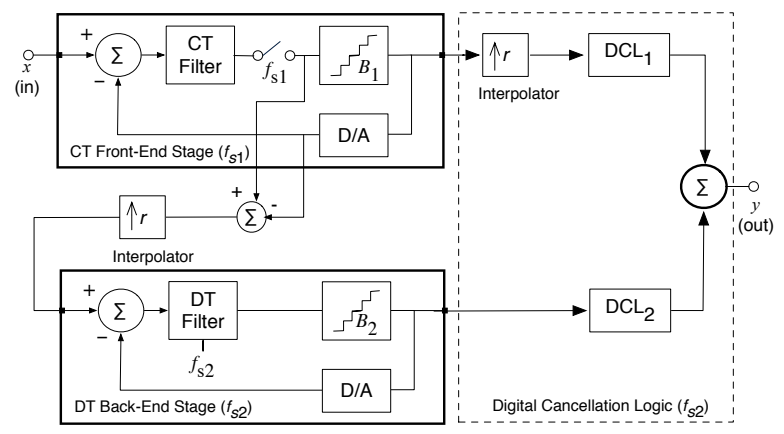

Figure 1. Block diagram of a conventional cascade MR H- $\Sigma \Delta \mathrm{M}$.

values of OSR in the (DT) back-end stages - where the speed requirements are less demanding than in the front-end ones [12]-[14].

The operation behind the modulator in Fig. 1 is conceptually the same as in a conventional monorate cascade $\Sigma \Delta \mathrm{M}$. All stage outputs are combined by the Digital Cancellation Logic (DCL) transfer functions so that ideally only the quantization error of the last stage remains, and it is shaped by a Noise Transfer Function (NTF) whose order, $L$, is the sum of the orders of all stages in the cascade, $L_{\mathrm{i}}$. Thus, assuming a linear model for the quantizers, it can be shown that the In-Band Noise (IBN) power at the output of an $N$-stage cascade MR $\Sigma \Delta \mathrm{M}$ is given by [12]:

$$
\mathrm{IBN}_{\mathrm{US}_{-} \mathrm{H}-\mathrm{MR}} \simeq \frac{\Delta^{2} \pi^{2 L}}{12(2 L+1)} \frac{1}{\mathrm{OSR}_{1}^{2 L_{1}} \mathrm{OSR}_{2}^{2 L_{2}} \ldots \mathrm{OSR}_{N}^{2 L_{N}}+1}
$$

where $\operatorname{OSR}_{i}=f_{\mathrm{si}} /\left(2 B_{\mathrm{w}}\right)$ stands for the oversampling ratio of the $i$-th stage, and $B_{\mathrm{w}}$ is the input signal bandwidth. Note that considering that all $\mathrm{OSR}_{i}$ are equal to OSR, the expression in (1) becomes the same as in the case of a monorate cascade $\Sigma \Delta \mathrm{M}$. The benefit of using lower values of OSR in the front-end CT stage is useful when the subsequent DT stages can operate at high enough OSRs. However, in spite of their less demanding speed requirements, the use of high values of OSR in the back-end stages is not practical when digitizing wideband signals, in the order of $10-100 \mathrm{MHz}$. In such a case,
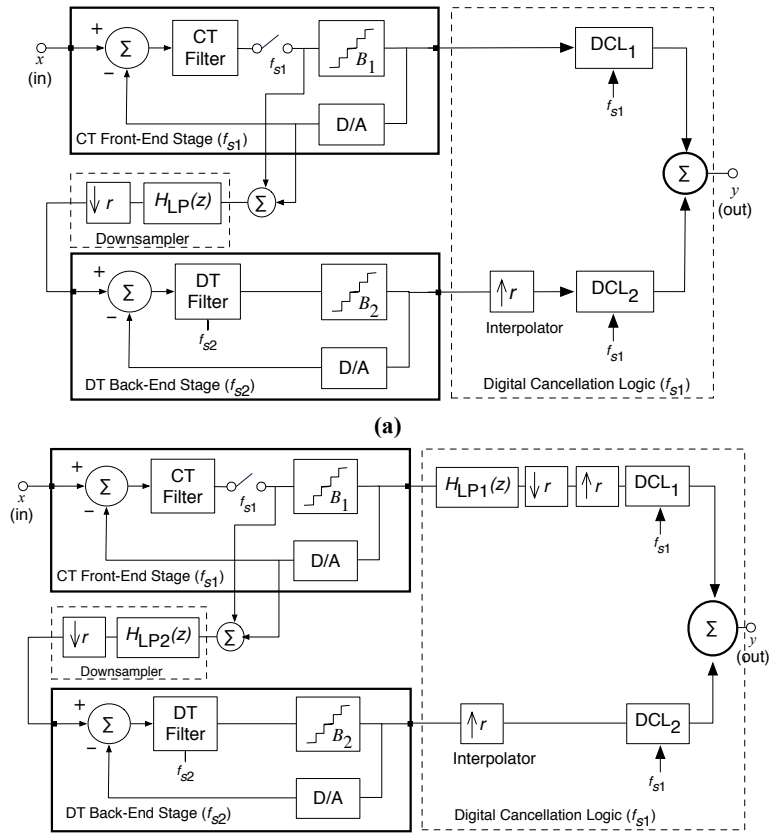

(b)

Figure 2. Proposed downsampling cascade (2-stage) MR H- $\Sigma \Delta \mathrm{Ms}$.

(a) Conceptual block diagram. (b) Including digital AAF. sampling rates in the order of $\mathrm{GHz}$ may be necessary in order to digitize such signals with low-medium effective resolutions (8-10 bits). In such a case, the use of back-end SC stages may not be practical because of the prohibitive values of the required Gain BandWidth (GBW) of the corresponding operational amplifiers. An alternative solution could be implementing a complete CT cascade MR $\Sigma \Delta \mathrm{M}$ [13]. However, this is less robust in practice as a consequence of the sensitivity of cascade CT- $\Sigma \Delta \mathrm{Ms}$ to circuit element tolerances, which is particularly critical in the case of nanometer CMOS implementations [4].

\section{Proposed MultiRate CASCADE H- $\Sigma \Delta \mathrm{Ms}$}

The mentioned problems of MR $H-\Sigma \Delta$ Ms, can be alleviated if the concept of multirating is redefined just in the opposite way as was conceived, i.e going from an upsampling multirate system - in which the front-end stage rate is increased in the subsequent stages - to a downsampling multirate system - in which the front-end (CT) stage operates at the highest sampling rate, thus benefiting from their potentially faster operation.

\section{A. From upsampling to downsampling $M R H-\Sigma \Delta M s$}

Fig. 2(a) shows a conceptual block diagram of the proposed downsampling cascade 2-stage MR $\mathrm{H}-\Sigma \Delta \mathrm{M}$. As the back-end (DT) stage operates at a lower rate than the front-end (CT) stage, an interstage (analog) AAF is needed in order to avoid aliasing. In practice, this block can be embedded in a SC implementation of the back-end integrators as suggested in [15]. Taking advantage of the DT-CT equivalence [2], the analysis of Fig.2(a) can be done starting from a complete DT- implementation of the MR $\Sigma \Delta \mathrm{M}$. The resulting hybrid CT/DT architecture can be easily synthesized by using a DT-to-CT transformation method [3][4]. Thus, assuming an ideal AAF transfer function, $H_{L P}(z)$ - i.e a complete cancellation of the aliased signal components - the $Z$-transform of the modulator output is given by:

$$
\begin{aligned}
& Y(z) \simeq \operatorname{STF}_{1}(z) D C L_{1}(z) X(z)+N T F_{1}(z) D C L_{1} E_{1}(z) \\
& -S T F_{2}\left(z^{1 / r}\right) H_{L P}(z) D C L_{2}(z) E_{1, \mathrm{DS}-\mathrm{US}}+N T F_{2}\left(z^{1 / r}\right) D C L_{2} E_{2, \mathrm{US}}
\end{aligned}
$$

where $r \equiv f_{s 2} / f_{s 1} \equiv \mathrm{OSR}_{2} / \mathrm{OSR}_{1} ; \operatorname{STF}_{1,2}(z)$ and $N T F_{1,2}(z)$ stand for the Signal- and Noise Transfer Functions of the first- and secondstages of the modulator, respectively; $E_{1,2}(z)$ represents the Ztransform of the quantization error; and the subscripts DS and US denote respectively that an operation of DownSampling and UpSampling have been applied to $E_{1,2}(z)$.

Note from (2) that, as a consequence of both DS and US operations performed over $E_{1}(z)$, this error cannot be completely cancelled since, theoretically, $E_{1}(z) \neq E_{1, \mathrm{DS}-\mathrm{US}}(z)$. This problem can be solved by applying the same signal processing to $E_{1}(z)$ in both the analog and the digital domain, as illustrated in Fig. 2(b). In this case, the $Z$-transform of the modulator output can be written as:

$$
\begin{aligned}
& Y(z) \simeq \operatorname{STF}_{1}(z) H_{L P 1}(z) D C L_{1}(z) X_{D S-U S}(z)+ \\
& +N T F_{1}(z) H_{L P 1}(z) D C L_{1} E_{1, \mathrm{DS}-\mathrm{US}} \\
& -\operatorname{STF}_{2}\left(z^{1 / r}\right) H_{L P 2}(z) D C L_{2}(z) E_{1, \mathrm{DS}-\mathrm{US}}+N T F_{2}\left(z^{1 / r}\right) D C L_{2} E_{2, \mathrm{US}}
\end{aligned}
$$

It is shown from (3) that $E_{1}(z)$ can be cancelled if

$$
\begin{aligned}
& H_{L P 1}(z)=H_{L P 2}(z) ; \\
& D C L_{1}(z)=\operatorname{STF}_{2}\left(z^{1 / r}\right) ; D C L_{2}(z)=\operatorname{NTF}_{1}(z)
\end{aligned}
$$

Assuming $\operatorname{STF}_{1,2}(z)=z^{-L_{1,2}}$ and $N T F_{1,2}(z)=\left(1-z^{-1}\right)^{L_{1,2}}$, it can be shown that the IBN power at the output of Fig. 2(b) is given by:

$$
\mathrm{IBN}_{2 \text {-stg_DS_H-MR }} \simeq \frac{\Delta^{2}}{12} \frac{\pi^{2\left(L_{1}+L_{2}\right)}}{r^{2 L_{2}} \mathrm{OSR}_{1}^{2\left(L_{1}+L_{2}\right)+1}\left[2\left(L_{1}+L_{2}\right)+1\right]}
$$

which results in the same expression as a monorate case when $r=1$. 


\section{B. Practical implementation of the proposed architecture}

Note from (4) that, in order to get a correct performance of the modulator in Fig. 2(b), a perfect matching is required between the analog and the digital versions of $H_{L P}(z)$, i.e $H_{L P 1}(z)$ and $H_{L P 2}(z)$, respectively. This matching requirement cannot be obtained in practice, thus resulting in a residual error that degrade the effective resolution of the modulator. This problem can be solved by using the proposed cascade MR H- $\Sigma \Delta$ M shown in Fig. 3(a). In this case, as the inter-stage (analog) AAF has been removed, the error signal, $E_{1}-$ which is sampled at the second-stage - will have aliased components at multiples of $f_{s 2}$. This can be expressed in the $Z$-domain as:

$$
E_{1}(z)=r \sum_{k=0}^{1 / r-1} E_{1}\left(z^{r} e^{j 2 \pi k r}\right)
$$

Note that, the aliased components are indeed additional quantization error components, and therefore, they can be also cancelled by properly designing the digital functions $H_{1,2}(z)$ for a given value of $r$. Thus, in the case of $r=1 / 2$ and $r=1 / 4$, it can be shown that both $E_{1}$ and its corresponding alias signal can be cancelled if the following condition is satisfied:

$$
H_{1}=H_{2}=\left\{\begin{array}{c}
\operatorname{NTF}_{1}(-z)(r=1 / 2) \\
\operatorname{NTF}_{1}\left(z e^{j \pi / 2}\right) \operatorname{NTF}_{1}(-z) N T F_{1}\left(z e^{j 3 \pi / 2}\right)(r=1 / 4)
\end{array}\right.
$$

Following the same procedure as in previous sections, it can be shown that the IBN at the output of the modulator in Fig. 3(a), is given by (5). However, on the contrary to the modulator in Fig. 2(b), the modulator in Fig. 3(a) does not need any additional analog block as compared to a conventional cascade $\mathrm{H}-\Sigma \Delta \mathrm{M}$. Moreover, the cancellation condition in (7) is completely performed in the digital domain, instead of the analog domain as in (4), thus making the implementation of Fig. 3(a) more robust in practice.

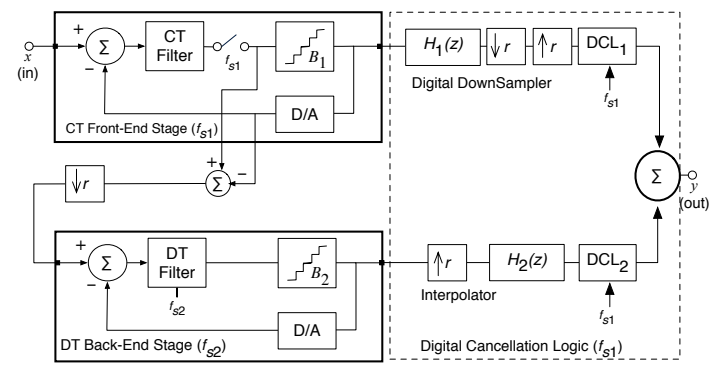

(a)

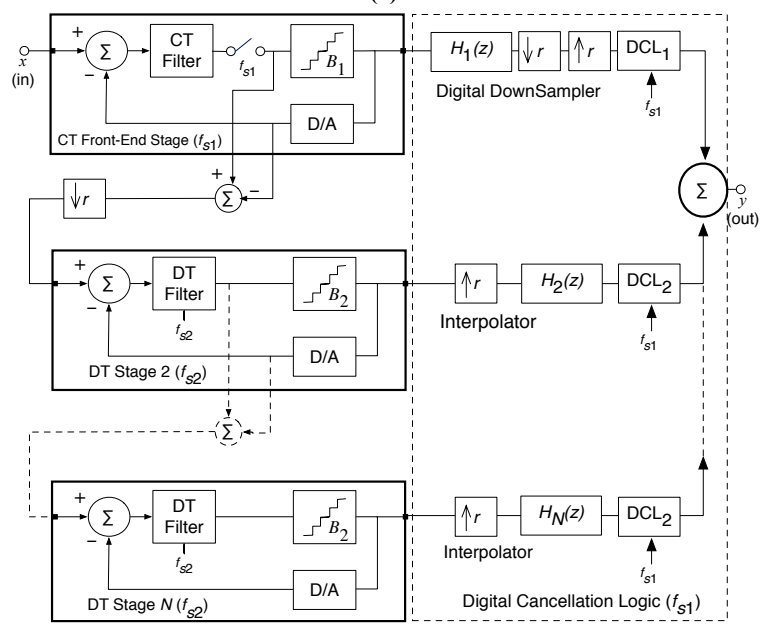

(b)

Figure 3. Proposed downsampling MR H- $\Sigma \Delta \mathrm{M}$ without analog AAF. (a) 2 -stage cascade. (b) Generic $N$-stage cascade topology.

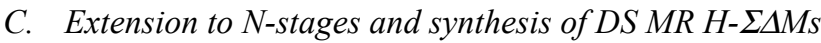

The concept of downsampling multirate $\mathrm{H}-\Sigma \Delta \mathrm{Ms}$ can be extended to a cascade of $N$ stages as illustrated in Fig. 3(b), where the front-end (CT) stage is sampled at $f_{s 1}$ and the remaining $N-1$ stages are sampled at $f_{s 2}<f_{s 1}$. Thus, in the more general case, the quantization error of the front-end stage (including its aliasing component) can be completely cancelled if $H_{i}(z)$ are all equal and satisfy the condition in (7). In such a case, and assuming $N T F_{1}(z)=\left(1-z^{-1}\right)^{L_{1}}$, eq. (7) transforms into

$$
H_{1}(z)=H_{2}(z)=\ldots=H_{N}(z)=\left(\sum_{k=0}^{1 / r-1} z^{-k}\right)^{L_{1}}
$$

and the IBN at the output of the modulator in Fig. 3(b) is given by:

$$
\mathrm{IBN}_{\text {DS_H-MR }} \simeq \frac{\Delta^{2} \pi^{2 L}}{12(2 L+1)} \frac{1}{r^{2\left(L-L_{1}\right)+1} \mathrm{OSR}_{1}^{2 L+1}}
$$

It is interesting to compare the above expression with the one obtained for a conventional multirate - given in (1). Thus, assuming that $\mathrm{OSR}_{i}=\mathrm{OSR}_{2}$ for $N \geq i>2$ it can be shown that:

$$
\frac{\mathrm{IBN}_{\text {DS_H-MR }}}{\mathrm{IBN}_{\text {US_H-MR }}} \simeq \frac{1}{r_{\mathrm{DS}}^{2\left(L-L_{1}\right)+1} r_{\mathrm{US}}^{2 L_{1}}}\left(\frac{\mathrm{OSR}_{2 \mathrm{US}}}{\mathrm{OSR}_{1 \mathrm{DS}}}\right)^{2 L+1}
$$

where $r_{\mathrm{DS}, \mathrm{US}}$ and OSR $\mathrm{ODS}, \mathrm{US}$ stand for the value of $r$ and $\mathrm{OSR}_{1,2}$ for the downsampling and upsampling MR $\mathrm{H}-\Sigma \Delta \mathrm{M}$ cases, respectively ${ }^{2}$. Assuming $\mathrm{OSR}_{1 \mathrm{DS}, 2 \mathrm{US}}=\mathrm{OSR}_{2 \mathrm{US}}$ and $r_{\mathrm{DS}}=1 / r_{\mathrm{US}}$, it is shown that expression in (10) reduces to $r_{\mathrm{DS}}^{2\left(2 L_{1}-L-1 / 2\right)}$, meaning that the proposed architecture is better than the conventional one if $L_{1}>L / 2+1$. However, if we consider $\mathrm{OSR}_{1 \mathrm{DS}}=k_{\mathrm{OSR}} \mathrm{OSR}_{2 \mathrm{US}}$ with $k_{\mathrm{OSR}}=1 / r_{\mathrm{DS}}=r_{\mathrm{US}}>1$, eq. (10) gives $r_{\mathrm{DS}}^{4 L_{1}}<1$. That is, the proposed architecture achieves a better effective resolution than a conventional multirate architecture if we assume that the maximum value of OSR, i.e the OSR of the CT stage, is larger than the maximum OSR of the conventional one. This condition is indeed very common in practice since the CT front-end stage is potentially faster than the SC ones.

\section{EXAMPLES AND SIMULATION RESUlTS}

As an illustration of the proposed methodology, Fig. 4 shows the block diagram of two DS MR cascade H- $\Sigma \Delta$ Ms: 2-1 (Fig. 4(a)) and 2-2 (Fig. 4(b)). In all cases, the modulators have been synthesized starting from a complete DT topology and applying a DT-to-CT transformation to the front-end stage. These modulators have been simulated using SIMSIDES, a time-domain behavioral simulator for $\Sigma \Delta \mathrm{Ms}$ [16] in order to verify the proposed methodology and architectures. As an illustration, Fig. 5 depicts the output spectra of the modulators in Fig. 4 for different values of $r$, showing the variation of the notch frequency associated to the multirate operation.

Fig. 6 represents IBN vs. OSR for different values of $r$ and the number of bits of the internal quantizers $(B)$, showing a good agreement between simulations and theoretical predictions. The impact of main circuit errors has been also considered. As an example, Fig. 7 shows a 500-sample Montecarlo simulation of the modulators in Fig. 4 for different values of $r$, considering a standard deviation of $1 \%$ in all analog coefficients. The IBN degradation is similar to the one obtained in conventional cascade $\Sigma \Delta \mathrm{Ms}$ [1].

\section{CONCLUSIONS}

A new concept of multirate hybrid (CT/DT) cascade $\Sigma \Delta \mathrm{M}$ has been presented. Contrary to conventional multirate architectures, the proposed $\Sigma \Delta \mathrm{M}$ topologies use the highest sampling rate at the (CT)

\footnotetext{
${ }^{2}$ Note from the definition of $r$ that $r_{\mathrm{DS}}<1$ and $r_{\mathrm{US}}>1$.
} 
front-end stage of the modulator instead of the back-end one. This approach exploits the potential capability of CT circuits to operate at higher frequencies, whereas relaxes the speed requirements of the DT parts of the modulator. The main drawback comes from the aliasing due to the implicit downsampling operation that takes place between the front-end and subsequent stages of the modulator. This problem is circumvented by properly designing the digital cancellation logic of the modulator, without needing any extra analog circuits as compared to conventional cascade $\Sigma \Delta \mathrm{Ms}$. The resulting $\mathrm{H}-\Sigma \Delta \mathrm{Ms}$ are potentially faster and more power efficient than conventional multirate modulators, being a promising approach to digitize $10-100 \mathrm{MHz}$ signals with medium-high resolutions.

\section{REFERENCES}

[1] R. del Río, F. Medeiro, B. Pérez-Verdú, J.M. de la Rosa and A Rodríguez-Vázquez: CMOS Cascade $\Sigma \Delta$ Modulators for Sensors and Telecom: Error Analysis and Practical Design. Springer, 2006

[2] M. Ortmanns and F. Gerfers: Continuous-Time Sigma-Delta A/D Conversion: Fundamentals, Performance Limits and Robust Implementations. Springer, 2006.

[3] H.K. Kwan et al.: "Design of Hybrid Continuous-Time Discrete-Time Delta-Sigma Modulators". Proc. of the 2008 IEEE Int. Symposium on

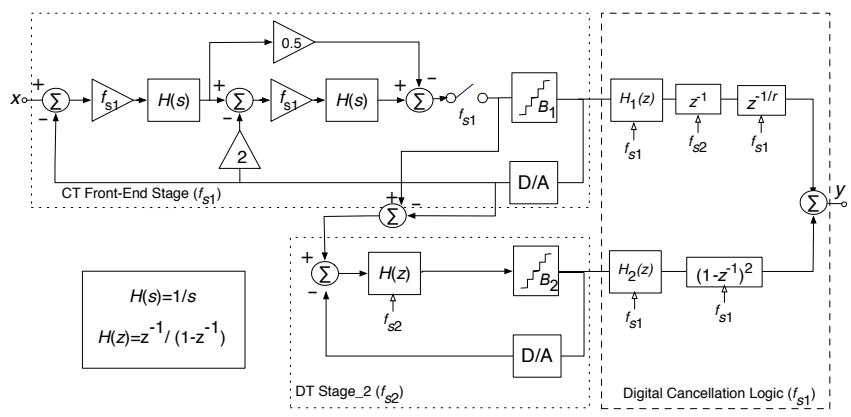

(a)

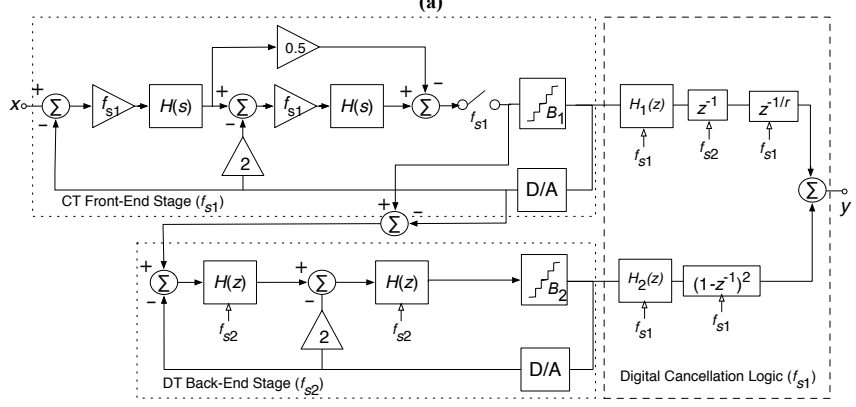

(b)

Figure 4. Examples of the proposed DS MR cascade $H-\Sigma \Delta \mathrm{Ms}$ :

(a) 2-1 architecture. (b) 2-2 architecture.

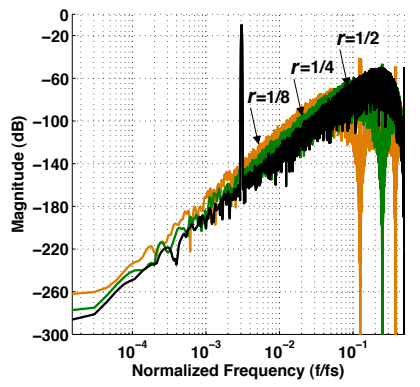

(a)

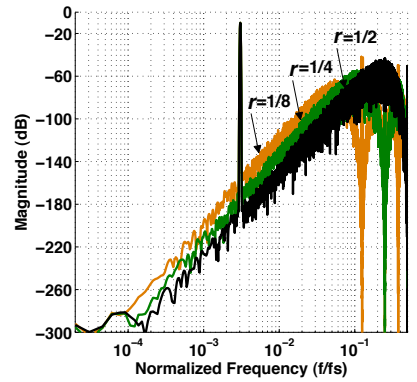

(b)
Figure 5. Output spectra of the modulators in Fig. 4: (a) 2-1. (b) 2-2.
Circuits and Systems (ISCAS), pp. 1224-1227.

[4] J.M. de la Rosa, A. Morgado and R. del Río: "Hybrid ContinuousTime/Discrete-Time Cascade $\Sigma \Delta$ Modulators with Programmable Resonation." Proc. of the 2009 IEEE Int. Symposium on Circuits and Systems (ISCAS), pp. 2249-2252.

[5] J.H. Shim et al.: "A Third-Order $\Sigma \Delta$ Modulator in 0-18- $\mu$ m CMOS With Calibrated Mixed-Mode Integrators". IEEE J. of Solid-State Circuits, pp. 918-925, April 2005.

[6] P. Morrow et al.: "A $0.18 \mu \mathrm{m}$ 102dB-SNR Mixed CT SC Audio-Band $\triangle \Sigma$ ADC". Proc. of the 2005 IEEE Int. Solid-State Circuits Conf., pp. 178-179, 2005.

[7] K. Nguyen et al.: "A 106dB SNR Hybrid Oversampling ADC for Digital Audio". Proc. of the 2005 IEEE Int. Solid-State Circuits Conf., pp. 176-177, 2005.

[8] B. Putter: "A 5th-Order CT/DT Multi-Mode $\Delta \Sigma$ Modulator". Proc. of the 2005 IEEE Int. Solid-State Circuits Conf., pp. 244-245, 2007.

[9] S. D. Kulchycki et al.: "A 77-dB Dynamic Range, 7.5-MHz Hybrid Continuous-Time/Discrete-Time Cascade $\Sigma \Delta$ Modulator." IEEE J. of Solid-State Circuits, pp. 796-804, April 2008.

[10] M.Y. Choi et al.: "A 101-dB SNR Hybrid Delta-Sigma Audio ADC Using Post Integration Time Control." Proc. of the 2008 IEEE Custom Integrated Circuits Conference (CICC), pp. 89-92.

[11] E.H. Dagher et al.: "A 2-GHz Analog-to-Digital Delta-Sigma Modulator for CDMA Receivers With 79-dB Signal-to-Noise Ratio in 1.23-MHz Bandwidth." IEEE J. of Solid-State Circuits, pp. 1819-1828, November 2004.

[12] F. Colodro et al.: "Multirate $\Sigma \Delta$ Modulators." IEEE Trans. on Circuits and Systems - II, vol. 49, pp. 170-176, March 2002.

[13] M. Ortmanns et al.: "Multirate Cascaded Continuous-Time $\Sigma \Delta$ Modulators." Proc. of the 2002 IEEE Int. Symposium on Circuits and Systems (ISCAS), pp. 2253-2256.

[14] M. H. Maghami and M. Yavari: "Multirate Double-Sampling Hybrid CT/DT Sigma-Delta Modulators for Wideband Applications." Proc. of the 2009 IEEE Int. Symposium on Circuits and Systems (ISCAS), pp. 2253-2256.

[15] A.C. Carusone and F. Maloberti: "An Anti-Aliasing Multi-Rate $\Sigma \Delta$ Modulator." Proc. of the 2009 IEEE Int. Symposium on Circuits and Systems (ISCAS), pp. 1329-1332.

[16] J. Ruiz-Amaya et al.: "High-Level Synthesis of Switched-Capacitor, Switched-Current and Continuous-Time $\Sigma \Delta$ Modulators Using SIMULINK-based Time-Domain Behavioral Models." IEEE Trans. on Circuits and Systems-I, vol. 52, pp. 1795-1810, Sept. 2005.

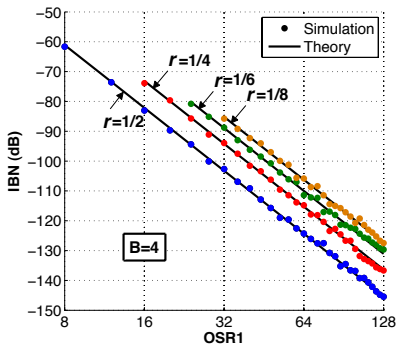

(a)

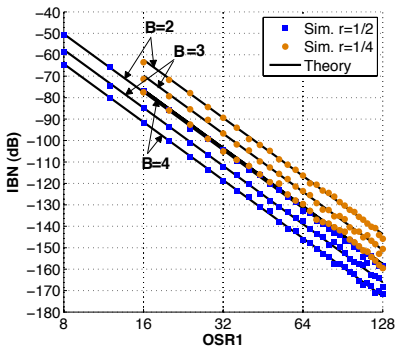

(b)
Figure 6. IBN vs. OSR for different values of $r$ and $B$ : (a) Modulator in Fig. 4(a). (b) Modulator in Fig. 4(b).

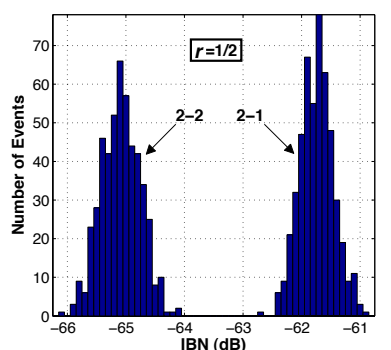

(a)

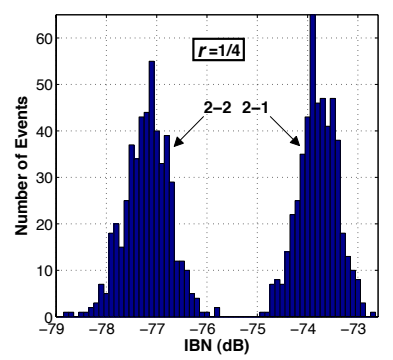

(b)
Figure 7. MonteCarlo Simulation of Fig. 4 for: (a) $\mathrm{OSR}_{1}=8, r=1 / 2$. (b) $\mathrm{OSR}_{1}=16, r=1 / 4$ 